\title{
The Influence of Grain Size of Dissimilar Welding between Carbon Steel and Stainless Steel on Ultrasonic Testing
}

\author{
Kwanruethai Sorn-ard*, Asa Prateepasen and Mai Noipitak \\ Department of Production Engineering, Faculty of Engineering, King Mongkut’s University of Technology Thonburi, Bangkok \\ ${ }^{*}$ Corresponding author
}

\begin{abstract}
The objective of this research is to study the ultrasonic attenuation effect on metallurgical properties of specimens. This study uses the various metallurgical grain sizes, using the ANSI1025 grade low carbon steel and ANSI316 grade stainless steel as base metals and shielded metal arc welding with E309L16 to create the discontinued area at the center of fusion zone and the fusion line. Both sides of the specimen are further tested with ultrasonic tests to determine the artificial discontinuity. It was found that different grain size has different effect from different specimens related to attenuation absorption rate. The result showed area created artificial discontinuity at the fusion line from ultrasonic testing only. The beneficial from this particular research work has lead to better understanding on the limitation of testing for the welding fusion zone between carbon steel and stainless steel, and will provide important information toward the selection of non-destructive testing (NDT) method for testing area of fusion zone discontinuity.
\end{abstract} size

Keywords-ultrasonic testing; stainless steel; carbon steel; grain

\section{INTRODUCTION}

The investigator has conducted an investigation into why metallic material of different properties have different residual strength once they have been welded together prior to forming product, this enable the remaining useful life to be predicted. The advantage of this research will be beneficial to casting, fabrication, auto industrial, construction, automobile spare parts and machinery parts. The investigation method is nondestructive testing of materials as no part of any product was destructed. The ultrasonic testing is a NDT test which will not alter the specimen in any form or shape and has been widely accepted and supported by The American society of mechanical engineers (ASME) codes and standards.

Principle of ultrasonic testing (UT) is using high-frequency sound waves energy generated from piezoelectric equipment. The sound was sent and propagated through the materials or specimens being tested. The limitation of this method is due to wave energy attenuation, reflections and scattering of ultrasonic wave energy. They traveled through metallurgical grain boundaries of the specimen. Nowadays, various testing methodology such as radiographic testing (RT) is quite popular and preferable but in many instances ultrasonic wave energy still being used, since radiographic testing is too cumbersome to setup and hence, in certain cases radiographic testing cannot be used totally due to curve and unable to access test area and ultrasonic testing will be used.

The investigations by previous researches were on carbon steel specimens only, same type material which did not differentiate between metallurgical grain size migrations from ultrasonic wave energy attenuation testing. [1,2] The metallurgical grain migration of two different thickness specimens $12.7 \mathrm{~mm}$ and $25.4 \mathrm{~mm}$ was studied, put together and then tested with the ultrasonic wave. The test was carried out, one specimen with respectively of dissimilar metal specimen for ultrasonic testing on this research to find the metallurgical grain migration and found out the bigger specimen take twice the time for ultrasonic testing energy penetration ratio 2:1 but did not mention how the metallurgical grain movement. [3] order to use ultrasonic testing, one required materials are acoustically isotropic i.e. carbon steel specimen. The inspection of weld steel normally has acoustic sound similar to the sound from carbon steel. The sound reflection will be the same direction. Fine-grained steel qualities meet these requirements. State of the ultrasonic inspection of stainless steel (austenitic) welds demands special procedures with component of specific qualifications for isotropic and anisotropic specimen. This is to demonstrate the dissimilar specimen welds testing. [4]

The objective of this research is to explain the effect of ultrasonic energy attenuation and study grain size influence of dissimilar property specimens from welding between carbon steel and stainless steel. Artificial discontinuities were created by technique and methodology from previous research [5] by creating an artificial discontinuity in welding of dissimilar specimens to study the variance of an artificial discontinuity at center of fusion zone was intentionally created to prove ultrasonic attenuation theory, effects of grain size and acoustic impedance by the difference of material types used.

\section{THEORIES}

Ultrasonic testing is one of the most conventional nondestructive testing methods. The ultrasonic theory complicates but its principle concept is simple. It can be applied to discontinuity detection, evaluation, material characterization and more. The sound energy is generated and propagated through the materials in the wave's form. If the discontinuity is in the wave path, part of sound energy will be reflected back from its surface. The display screen will show 
in the significant echo between initial pulse and back wall echo. This signal results can be expressed to inform about the location, orientation and type of discontinuity.

\section{A. Transmission And Refraction At An Interface [6]}

From the equation of ultrasonic velocity, the equation of longitudinal sound velocity $(V)$ can be determined as in (1).

$$
V=\sqrt{E / \rho} \text { or } E=\rho V^{2}
$$

where $\rho$ is the density of material, $E$ is Young's modulus.

When the unit of stress is force per area, the equation can be represented by the acoustic pressure $(p)$, the acoustic impedance $(\boldsymbol{Z})$ can be calculated as in (2).

$$
Z=\frac{\rho}{V_{\text {particle }}}=\frac{\rho V^{2} \frac{\partial u}{\partial x}}{\frac{\partial V}{\partial t}}=\rho V^{2} \frac{\partial t}{\partial x}=\frac{\rho V^{2}}{V^{2}}=\rho V
$$

The propagating ultrasonic wave or ultrasonic energy will be changed when it encounters a boundary such as grain boundaries. When materials have different specific of acoustic impedance, discontinuity type can be distinguished. Changing in the wave as it encounters an interface possible to take transmission, wave propagation, refraction, and mode conversion.

The incident wave intensity reflection can be derived because particle velocity and local particle pressure must be continuous across the boundary. The fraction of the incident wave intensity that is reflected can be calculated as in (3).

$$
E=\frac{z_{2}-z_{1}}{Z \cdot+z_{2}}
$$

when $R$ is the reflection wave coefficient, $Z_{1}$ is the acoustic impedance of material 1 and $Z_{2}$ is the acoustic impedance of material 2, respectively.

The transmission coefficient $(T)$ is calculated by simply subtracting the reflection coefficient from one or calculated as in (4). The reflected and transmitted energy is illustrated in Figure 1.

$$
T=\frac{2 Z_{2}}{\left(Z_{1}+z_{2}\right)}
$$

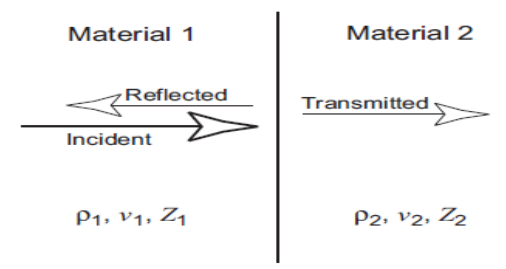

FIGURE I. REFLECTION BETWEEN THE NO. 1 AND NO. 2 MEDIUM

\section{B. Energy Attenuation [7]}

The sound energy traveled through a medium will be decreased with distance. In idealized materials, sound pressure is only reduced by the spreading of the wave. However, the sound energy can be scattered and absorbed by material. Scattering is the reflection of the sound in directions other than its original direction of propagation. Absorption is the conversion of the sound energy to other forms of energy such as heat. The combined effect of scattering and absorption is referred as the attenuation. Ultrasonic attenuation is the decay rate of the wave as it propagates through material.

The amplitude or sound energy change of a decaying plane wave can be expressed as in (5).

$$
A=A_{0} \exp (-\omega x)
$$

where $A$ and $A_{0}$ are the amplitude or sound energy after propagation into the medium in $x$ direction and the before entering medium, respectively. ${ }^{a}$ is the attenuation coefficient of the sound wave, $a=\frac{1}{x} \cdot \ln \frac{A}{A_{x}}$. $x$ is the propagation distance of the sound wave.

\section{Attenuation from Grain Size [7]}

The energy of sound wave is continuously reduced when the sound wave passes grain boundaries caused reflecting and scattering phenomenon. The energy of sound wave is diverted from the main wave into waves traveling in the different directions. This process is also called the attenuation of sound energy. The relationships of attenuation coefficient $(\alpha)$, wave length $(\lambda)$ and grain size $(D)$ are shown below.

$$
\begin{array}{ll}
\text { Case I }: \lambda \gg D & : \alpha_{p}=V f^{4} \\
\text { Case II }: \lambda \leq D & : \alpha_{p}=D f^{2} \\
\text { Case III }: \lambda<\infty< & : \alpha_{d}=1 / D
\end{array}
$$

where $f$ is the frequency of the ultrasonic wave.

\section{EXPERIMENTAL SETUP}

This research studied the testing of specimen between carbon steel and stainless steel on ultrasonic testing, which have the artificial discontinuity setups shown as following.

\section{A. Preparation of specimen}

A specimen of carbon steel ANSI1025 and stainless steel ANSI316 respectively of dissimilar property were tested for this particular research. The specimens have been cut $100 \mathrm{~mm}$ width, $150 \mathrm{~mm}$ length and $10 \mathrm{~mm}$ thickness. Both specimens have been cut into single v-groove $60^{\circ}$ angle then aligned them on side by side where the root face is equal to $3 \mathrm{~mm}$ wide and the root cap distance equal $3 \mathrm{~mm}$. 


\section{B. Creation of Artificial Discontinuity}

The lack of fusion on carbon steel in the first specimen and on stainless steel in the second specimen. In the third specimen, drilling a hole $1 \mathrm{~mm}$ diameter and $20 \mathrm{~mm}$ deep in the fusion zone, by this method will simulate the discontinuity in fusion zone such as the porosity and slag inclusion. The discontinuity locations as per Figure 2

Case No.1 The first specimen was prepared by placing one carbon steel plate and one stainless steel plate side by side and then weld both plates together. Then the lack of fusion in fusion line on the carbon steel side was created. After that the specimen was welded in slanting position with shielded metal arc welding (SMAW) with 309L-16 size $3.2 \mathrm{~mm} \times 350 \mathrm{~mm}$. Finally, the overlay welding occurred over the discontinuity until it is filled completely.

Case No.2 The second specimen was prepared using a technique as mentioned in case no.1. However in case no.2 the discontinuity of the lack of fusion is on stainless steel side of specimen.

Case No.3 The third specimen was prepared by using a technique as mentioned in case no.1 and then the discontinuity at center of fusion zone was created to simulate a porosity and slag inclusion by drilling a hole $1 \mathrm{~mm}$ diameter, $20 \mathrm{~mm}$ depth.

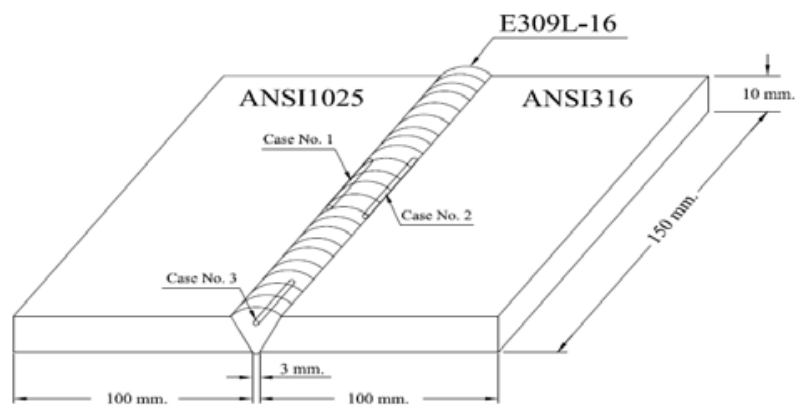

FIGURE II. THE OVERLAY WELD COVERING UP THE DISCONTINUTIT LOCATIONS

\section{Influence of Gain Size on Ultrasonic Testing}

The artificial discontinuity will be confirmed by using radiographic testing method. In order to test the influence of grain size on ultrasonic testing, ultrasonic probe frequency of 4.0 $\mathrm{MHz}$ was mounted on Ultrasonic flaw detector model USM Go + with probe angle $45^{\circ}, 60^{\circ}$ and $70^{\circ}$, respectively. Then the probe was placed on the specimen to inspect the artificial discontinuity and moved to the different locations and sides. The amplitude of ultrasonic wave in each different locations and sides were recorded.

\section{Microstructure and Grain Size Measurement}

The test specimens, $10 \mathrm{~mm} \times 30 \mathrm{~mm}$, were prepared and polished by sand paper to test the microstructure and grain size as shown in Figure 7. The microstructures of 5 spot, carbon steel, fusion line of carbon steel, fusion zone, fusion line of stainless steel, stainless steel as Figure 8, were tested by microscope model OLYMPUS BX51N. The grain sizes were measured by using MetImage V4.11 program, according to ASTM E1382 standard. [9]

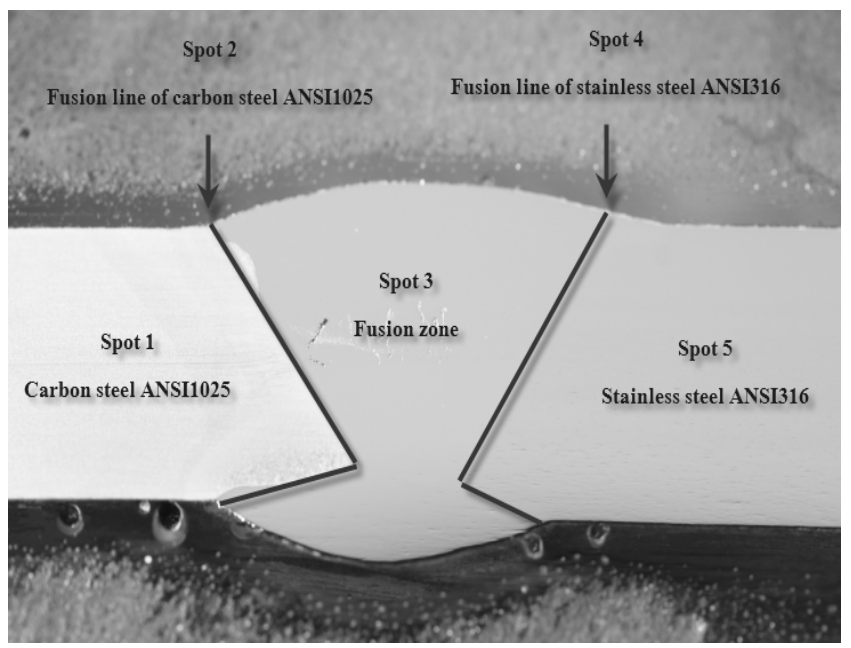

FIGURE III. THE MICROSTRUCTURE SPOT

\section{EXPERIMENTAL RESULT AND DISCUSSION}

\section{A. Artificial Discontinuity in Welded Specimen}

The creations of discontinuity in 3specimens with the result of non-destructive were tested by the radiograph testing as following.

The first discontinuity created the lack of fusion on carbon steel side $25 \mathrm{~mm}$ lengths

The second discontinuity created the lack of fusion on stainless steel side $30 \mathrm{~mm}$ lengths

The third discontinuity created the complete weld area and then creation of drilling a center hole in the fusion area $1 \mathrm{~mm}$ diameter, 20mm depth

\section{B. Result of Ultrasonic Testing}

The ultrasonic testing methodology comprised of sending out high frequency sound by showing the result on the monitor with the number of any parameter and DAC (Distance Amplitude Corrector) as the line for analyzing the result and aspect of signal. If the ultrasonic wave energy meets the discontinuity, it will reflect back to the monitor. And if the ultrasonic wave energy is not reflected that meaning there is no any discontinuity found on the specimen. As the scanning sensitivity was carried out to widen the search and increase the signal band size for easy visibility. And if the reflection signal was found, before making any decision from the test may require to reduce the power to match with the setting sensitivity value.

\section{1) Lack of fusion in carbon steel}

The result showed the discontinued signal on the monitor of the lack of fusion in the carbon steel side testing. On the other hand when testing with the lack of fusion in stainless steel side, there is no any signal on the monitor see the below Figure 4 and Table 1 tabulated detail. 
TABLE I. LACK OF FUSION IN CARBON STEEL

\begin{tabular}{|c|c|c|c|c|c|c|c|c|c|c|}
\hline \multirow{2}{*}{ Probe angle } & \multirow{2}{*}{ Index [mm] } & \multirow{2}{*}{ Sound path [mm] } & \multirow{2}{*}{ Depth[mm] } & \multicolumn{3}{|c|}{ Location [mm] } & \multicolumn{2}{|c|}{ Size $[\mathrm{mm}]$} & \multirow{2}{*}{ Amplitude } & \multirow{2}{*}{ Type of Discontinuity } \\
\hline & & & & $X$ & $Y$ & $Z$ & $C S$ & $S S$ & & \\
\hline $45^{\circ}$ & 13 & 20.86 & 5.25 & 18.75 & 50 & +4 & 22 & 一 & Upper 100\% & Lack of fusion \\
\hline $60^{\circ}$ & 13 & 27.37 & 6.31 & 27.7 & 50 & +4 & 22 & 一 & Upper $100 \%$ & Lack of fusion \\
\hline
\end{tabular}

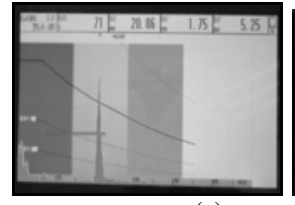

(a)

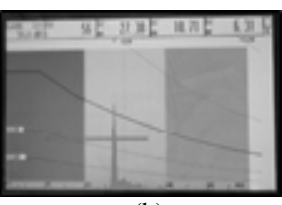

(b)

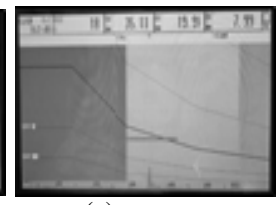

(c)

\section{2) Lack of fusion in stainless steel}

It shows the discontinued signal on the stainless steel testing, however, there is no any signal in monitor during the carbon steel testing see the result of signal below Figure 5 and Table 2 tabulated detail.

FIGURE IV. THE AMPLITUDE OF ULTRASONIC SIGNAL, CARBON STEEL (A) PROBE ANGLE $45^{\circ}$, (B) PROBE ANGLE $60^{\circ}$ AND (C) PROBE ANGLE $70^{\circ}$

TABLE II. LACK OF FUSION IN STAINLESS STEEL

\begin{tabular}{|c|c|c|c|c|c|c|c|c|c|c|}
\hline \multirow{2}{*}{ Probe angle } & \multirow{2}{*}{ Index[mm] } & \multirow{2}{*}{ Sound path [mm] } & \multirow{2}{*}{ Depth[mm] } & \multicolumn{3}{|c|}{ Location [mm] } & \multicolumn{2}{|c|}{ Size $[\mathrm{mm}]$} & \multirow{2}{*}{ Amplitude } & \multirow{2}{*}{ Type of Discontinuity } \\
\hline & & & & $\boldsymbol{X}$ & $\boldsymbol{Y}$ & $Z$ & CS & $S S$ & & \\
\hline $45^{\circ}$ & 13 & 22.63 & 4 & 17 & 75 & +1 & 一 & 24 & Upper 50\% & Lack of fusion \\
\hline $60^{\circ}$ & 13 & 29.78 & 5.11 & 26.79 & 75 & +1 & 一 & 24 & Upper 50\% & Lack of fusion \\
\hline
\end{tabular}

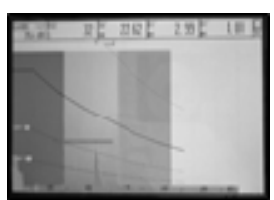

(a)

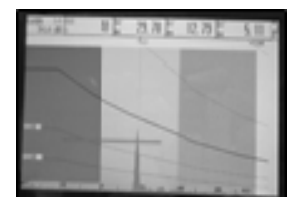

(b)

(c)

FIGURE V. THE AMPLITUDE OF ULTRASONIC SIGNAL, STAINLESS STEEL (A) PROBE ANGLE $45^{\circ}$, (B) PROBE ANGLE $60^{\circ}$ AND (C) PROBE ANGLE $70^{\circ}$

\section{3) Discontinuity at center of fusion zone}

The discontinuity in specimen was simulated by creating the hole in the center of fusion area $1 \mathrm{~mm}$ diameter, $20 \mathrm{~mm}$ depth, using ultrasonic testing to see if any notable discontinuity from each side wall of the hole is detectable. The result showed no discontinuity occurs.

\section{Microstructure and Grain Size}

The microstructures and grain size of both materials in fusion line and fusion zone were investigated under microscope photo by MetImage V4.11 program. The examples of microstructure are shown in Figure 6.

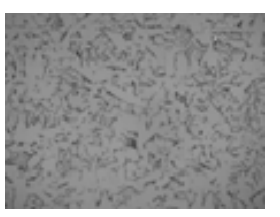

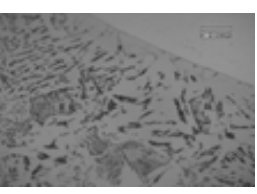

(c) (a)

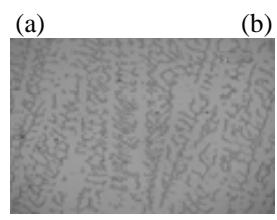

(d)

(b)
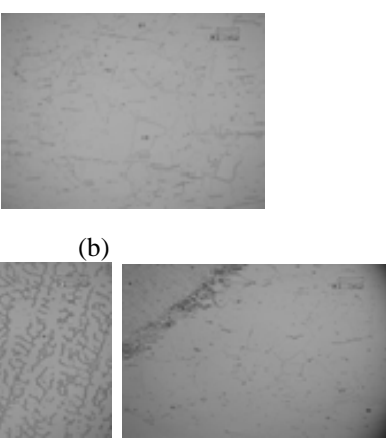

(e)
FIGURE VI. THE EXAMPLES OF MICROSTRUCTURE (A) CARBON STEEL, (B) STAINLESS STEEL, (C) FUSION LINE OF CARBON STEEL, (D) FUSION ZONE (WELD METAL) AND (E) FUSION LINE OF STAINLESS STEEL

From the Figure 6(a), the microstructure of carbon steel contains the Widmanstatten ferrite phase and pearlite phase. The grain size evaluation can be determined by using ASTM E 1382 standard [9] and the grain size is 8.4 The microstructure of stainless steel in Figure. 6(b) contains the austenite phase with carbide and grain size is 10 . The microstructure of fusion line of carbon steel in Figure 6(c) shows the result of overlay of welding between carbon steel with filler E309L-16 to create 
a new grain size of the Widmanstatten ferrite phase and pearlite phase and grain size is 16.8. Fusion zone in Figure 6(d) welded by E309L-16 electrode shows dendrite structure with ferrite phase and carbide in austenite phase and the grain size is 33.6. The microstructure of fusion line of stainless steel in Figure 6(e) shows carbide in austenite phase and the grain size is 23.8 .

\section{CONCLUSION}

The experimental result of the lack of fusion in carbon steel, the lack of fusion in stainless steel and the discontinuity at center of fusion zone by simulating a porosity and slag inclusion were carried out and the size difference of grain will affect the ultrasonic wave energy observed. The study was found that grain size has the wave energy attenuation. From the theory, the wave energy attenuation coefficient of fusion zone with stainless steel and carbon steel varied significantly due to material grain size differences. The study was found that the ultrasonic wave energy attenuation coefficients for carbon steel and stainless steel materials are 38 and 64 times respectively.

The ultrasonic wave energy attenuation in fusion zone is higher than other part of specimen so the test cannot show any result in fusion zone. The ultrasonic testing in stainless steel fusion zone area shows different results from the radiograph and very different from the result of the lack of fusion in carbon steel. As the size of grain in fusion zone in stainless steel has a bigger size contributing to a higher ultrasonic wave energy attenuation coefficient as compared to carbon steel will enlarge the error of result.

\section{ACKNOWLEDGEMENT}

The research team would like to take this opportunity to thank the King Mongkut's University of Technology for financial support on this research. The team would like to take Experteam Co., Ltd. for greatly contribute to provide material specimen, welding test facility, all related material preparation. We would like to thank Siwa Testing Inspection \& Consulting Co., Ltd. in providing the ultrasonic testing and radiographic testing equipment to carry on the research experiment.

\section{REFERANCES}

[1] Khwansri, B. and Prateepasen, A. Effect of grain size and rolling direction on velocity and attenuation of ultrasonic wave. In IE Network Conference 2012, Phetchaburi, Thailand, 2012. (in Thai)

[2] Bouda, A. B., Lebaili, S., and Benchaala, A. Grain size influence on ultrasonic velocities and attenuation. Journal of NDT\&E international 36(1): 1-5, 2003

[3] Smith, J. H. Ultrasonic evaluation of dissimilar-metal transition joint welds, Oak Ridge, Tennessee, 1979, pp.1-43.

[4] Bulavinov, A., Kröning, M., and Walte, F. (2007) Ultrasonic inspection of austenitic and dissimilar welds. In Conferencia Panamericana d END Buenos Aires-Octubre 2007, Buenos Aires, Argentina, 2007.

[5] Potidokmai, N. and Prateepasen, A. Manufacturing of lack of rusion in practical-training-specimen for ultrasonic testing. In $5^{\text {th }}$ Naresuan research conference, Phitsanulok, Thailand, 2009. (in Thai)

[6] Shull, J. P. and Tittmann, R. B. Ultrasound. In Nondestructive evaluation theory, techniques, and application (Shull, J. P., ed.), Marcel Dekker, Inc., New York, 2002, pp. 63-101.
[7] Prateepasen A. Ultransonic testing: UT. In Non-destructive testing in welds and researches (Prateepasen A., ed.), Chulalongkorn University Publishing Ltd., Bangkok, 2004, pp. 185-233. (in Thai)

[8] Fitting, W. D. and Adler, L. Ultrasonic spectral analysis for nondestructive evaluation, Plenum, New York, 1981, pp. 128-132.

[9] ASTM Standard E1382-97, "Standard test methods for determining average grain size using semiautomatic and automatic image analysis," ASTM International, West Conshohocken, PA, 2010,DOI: 10.1520/E138297R10, www.astm.org 